\title{
Quantum Mechanical Study on the $\pi$ - $\pi$ Stacking Interaction and Change in Conformation of Phenolic Systems with Different Intermolecular Rotations
}

\author{
Ibrahim Ali, Shruti Sharma, Bipul Bezbaruah* \\ Department of Applied Sciences, Gauhati University, Guwahati, India \\ Email: `bipulbezbaruah@gmail.com
}

How to cite this paper: Ali, I., Sharma, S. and Bezbaruah, B. (2018) Quantum Mechanical Study on the $\pi-\pi$ Stacking Interaction and Change in Conformation of Phenolic Systems with Different Intermolecular Rotations. Computational Chemistry, 6, 71-86.

https://doi.org/10.4236/cc.2018.64006

Received: July 26, 2018

Accepted: October 19, 2018

Published: October 22, 2018

Copyright $\odot 2018$ by authors and Scientific Research Publishing Inc. This work is licensed under the Creative Commons Attribution International License (CC BY 4.0).

http://creativecommons.org/licenses/by/4.0/

\section{Open Access}

\begin{abstract}
Aromatic systems like phenol, diphenol, cyano benzene, chloro benzene, aniline etc shows effective $\pi-\pi$ stacking interactions, long range van der Waals forces; ion- $\pi$ interactions etc. and these forces of interactions play an crucial role in the stability of stacked $\pi$-dimeric system. On the other hand, substituents and conformational change in the stacked dimmers of aromatic system may also change the stability of different stacked dimers. In this current study, stacked phenolic dimmers (both phenol and diphenol) have been taken for investigation of the stacking $\pi$ - $\pi$ interaction. But, the stacking interactions are also greatly affected by the conformational change with internal rotation (i.e. dihedral angle, $\varphi$ ) between the stacked dimers. It is generally accepted that larger basis sets are required for the highly accurate calculation of interaction energies for any stacked aromatic models. But, it has recently been reported that M062X/6-311++G(d,p) basis set is effectively better than that of B3LYP/6-311++G(d,p) for determining the interaction energies for any kind of long range interaction in aromatic systems. Therefore, all the calculations were carried out by using M062X/6-311++G(d,p) basis set. However, in most of the cases the calculated $\pi$ - $\pi$ stacking interaction energies show almost same result for both DFT and $a b$ initio methods.
\end{abstract}

\section{Keywords}

Phenolic System, $\pi-\pi$ Stacking, B3LYP, M062X

\section{Introduction}

Non covalent interactions in phenolic dimers refer $\pi-\pi$ staking interaction, 
which is basically an attractive interactive force between the aromatic rings. Just like an electrostatic interaction, where a region of negative charge interacts with a positive charge, the electron rich $\pi$-system can interact with a metal (cationic or neutral) or an anion with $\pi$-system [1] [2] [3]. This interaction is very useful in self-organisation and stabilization of aromatic molecules or any other $\pi$-systems [4] [5] [6]. However, phenolic systems are frequently associated by intermolecular $\mathrm{H}$-bonding. The importance of the $\pi-\pi$ stacking interaction has been repeatedly stressed in many fields of chemistry and structural biology [7] [8] [9]. The best known example is the stacking interaction between Nucleic acid (AT and GC base pairs) in DNA/RNA chains, where H-bonds between the base pairs are responsible for the stability and conformational arrangement of nucleic acid chain [10] [11] [12] [13]. Computational and Theoretical techniques such as $a b$ initio or DFT methods are widely used for the calculation of non-covalent interactions [14] [15]. Although, the gas phase calculations play a major role for determining the $\pi$ - $\pi$ stacking interactions but the solvent effect or polarity might change the stability of such stacked molecular systems [16] [17] [18] [19] [20].

In this current research work, we have performed a comprehensive study on the $\pi-\pi$ stacking interaction energy of phenolic systems by using DFT methods. During the last few years, there has been a significant progress in the method of calculating interaction energies in many aromatic complexes [21] [22]. It has been observed that in some cases the stacking stabilization energies computed by DFT method with extended basis sets is over estimated especially for stacked structures [23] [24] [25]. Phenol is one of the simplest aromatic systems and it can directly interact with each other through $\pi$ - $\pi$ stacking to form dimer, trimer, etc. as similar to water molecules. It is very complicated to calculate the $\pi-\pi$ stacking interaction more precisely for the sterically hindered phenols and other phenolic systems with bulky groups [26].

In this current research comprehensive computational investigations have been carried out for determining the actual $\pi-\pi$ stacking interactions of different stacked dimers of phenolic systems such as phenol-phenol, diphenol-diphenol and phenol-diphenol models. The $\pi$ - $\pi$ stacking interactions in the stacked models of phenolic dimers directly effect on the proper molecular geometry, atomic charge distribution, spin densities and intermolecular rotation or dihedral angles of the stacked models [27] [28] [29]. The stability of different eclipsed and staggered models of such stacked phenolic dimers has been investigated by using quantum mechanical methods. However, new function such as M062X based upon the density functional theory (DFT) gives an important area of computational research to investigate the non-covalent interactions. An investigation has also been carried out for determining the stacking interactions of phenol and diphenol in the different conformation with dihedral angles. It has been observed that $\pi-\pi$ stacking interactions in aromatic systems are greatly affected by change in different molecular rotation and it also depends on the electron cloud density of the stacked model; hence the change in electron cloud density on the stacked models was also calculated. For some stacked models of phenolic sys- 
tems, substituents on the phenol may also play an important role in the stability of stacked models. Therefore, in this current work $\pi-\pi$ stacking interactions of stacked phenolic dimer with different conformational change and intermolecular rotation (or dihedral angle), electron charge density, compatibility to the other substituents and effect of mixed phenolic stacked models would be investigated.

\section{Methodology}

\subsection{Computational and Theoretical Method}

Density functional Method (DFT) of calculation found to be very effective for studying non-bonded long range interactions in aromatic system. Nowadays, these methods have become an important tool for calculating all kinds of $\pi$ - $\pi$ stacking interaction. The Gaussian09 program has been widely used for DFT method to calculate the interaction energies of the molecular systems. The use of DFT is known for the applicability in medium and large molecular system. It is limited in system where dispersion part is considered as the dominant part and in that case the calculated interaction energy values are always under estimated. On the other hand, if a reasonably larger basic set is used then these method of calculations account well for determining the interaction energies as well as the electronic correlation energy of the molecules in the gas phase. The commonly used B3LYP method fails to predict dispersion energy, therefore in such cases inclusion of dispersion energy is very difficult for a large system. All the electron correlation energies for phenol and diphenol system have been calculated by DFT level of theory. All the geometrics of the studied molecules were optimized by using M062X/6-311++G(d,p), basic sets. All single point calculation were carried out by using M062X, B3LYP with $6-311++\mathrm{G}(\mathrm{d}, \mathrm{p})$ basis set. But, the minimized stacked structures were obtained by M062X method which shows the most favoured geometry. All the calculations were performed with the Gaussian 09 software Package and the visualization was done by GaussView5.0 [30].

The interaction energy for the stacked geometry can directly be calculated by the following equation:

$$
\text { Interaction energies }=\mathrm{E}_{\mathrm{st}}-2 \times \mathrm{E}_{\mathrm{us}}
$$

Here, $\mathrm{E}_{\mathrm{st}}=$ energy of stacked model, $\mathrm{E}_{\mathrm{us}}=$ energy of unstacked model.

\subsection{Construction of Models}

Phenols are generally packed with its neighbours through weak vander waals forces to form dimer, trimer tetramer etc. Generally in normal conditions, one phenol ring could directly stack parallelly with other phenol ring by different ways either in eclipsed or staggered conformation. The eclipsed conformation of stacked phenolic dimers show exact sandwich form at a dihedral angle of $0^{\circ}$. On the other hand, the staggered conformation for stacked phenolic dimers may also suitably stacked at different dihedral angles, viz. $60^{\circ}, 120^{\circ}$ and $180^{\circ}$. The individual models in a stacked conformation of stacked phenolic dimers are sepa- 
rated at a fixed vertical separation of $3.6 \AA$, which is found to be the most favoured separation between two phenol rings. The $-\mathrm{OH}$ group of the phenol ring also plays an important role in the stability of stacking interaction in the stacked phenolic dimers. Therefore, during the construction of stacked models, $-\mathrm{OH}$ group of the two phenol rings may be placed either in same or opposite direction. Here, we have constructed the stacked models to study the stacking interactions of following phenolic systems:

\section{Result and Discussion}

In this investigation, the $\pi-\pi$ stacking interactions of phenolic dimers such as Phenol-Phenol, Diphenol-Diphenol and Phenol-Diphenol, have been studied for different conformations in gas phase. All the phenolic systems were optimized by using DFT method with M062X/6-311++G(d,p) basis set (Figure 1). All the conformations for stacked phenolic systems were prepared with different dihedral angles such as $0^{\circ}, 60^{\circ}, 120^{\circ}$ and $180^{\circ}$ (Figure 2). But, for phenol-diphenol and diphenol-diphenol stacked models $0^{\circ}$ is equivalent to $180^{\circ}$ and $60^{\circ}$ is equivalent to $120^{\circ}$, therefore we prepared models only for $0^{\circ}$ and $60^{\circ}$ dihedral angles. Phenolic stacked models with dihedral angle $0^{\circ}$ was considered as the eclipsed conformation, which was prepared by placing one phenol ring parallely over the other ring with an internal separation of $3.6 \AA$ (Figure 3 ). On the other hand, dihedral angles $60^{\circ}, 120^{\circ}$ and $180^{\circ}$, were considered as staggered conformation. During the construction of stacked phenol-phenol model, one phenol ring has been horizontally shifting along X, Y or Z-axis (from positive to negative end), keeping the other ring at constant position. This process was carried out to get the most favored minimized stacked model with minimum repulsion as well as the highly repulsive stacked model. In this case, the horizontal shifting for the stacked model was investigated along X-axis from -3 to $+3 \AA$. Similarly, all the staggered conformations for the stacked models of phenol-phenol stacking systems were also prepared with dihedral angles $60^{\circ}, 120^{\circ}$ and $180^{\circ}$ respectively.

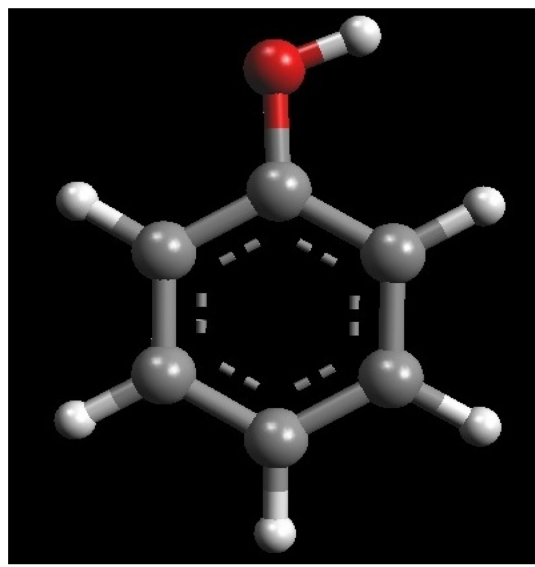

(a)

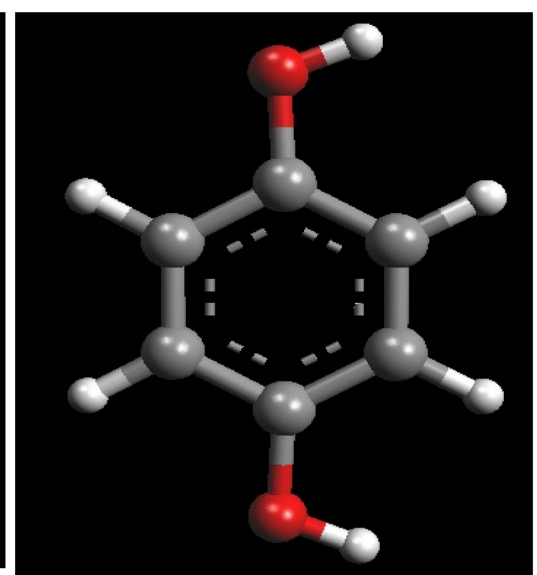

(b)

Figure 1. Optimized models for (a) Phenol and (b) Diphenol. 


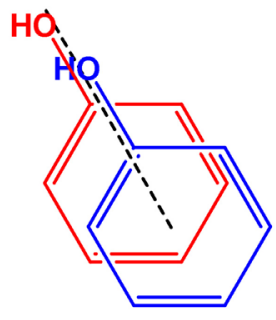

$0^{\circ}$

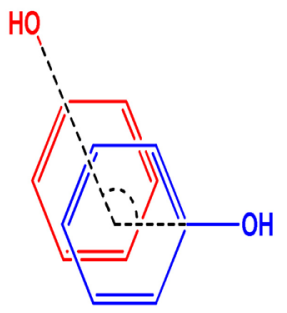

$120^{\circ}$

HO

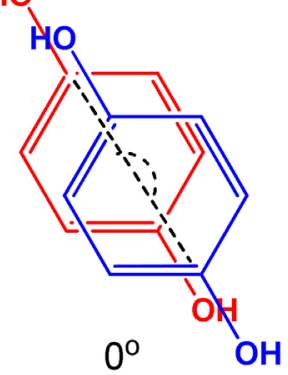

HO

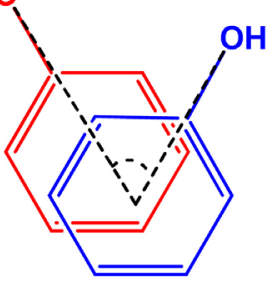

$60^{\circ}$

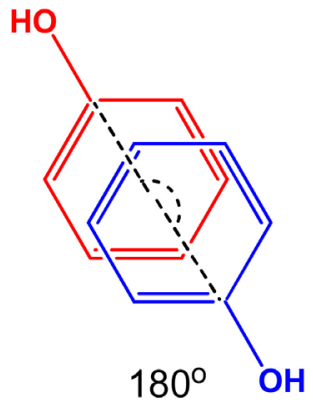

(a)

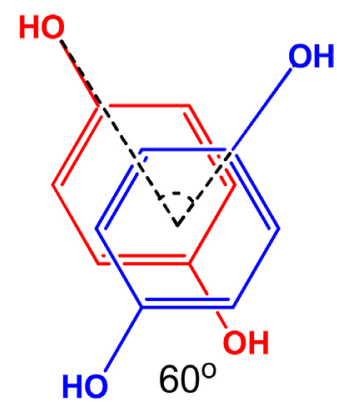

(b)
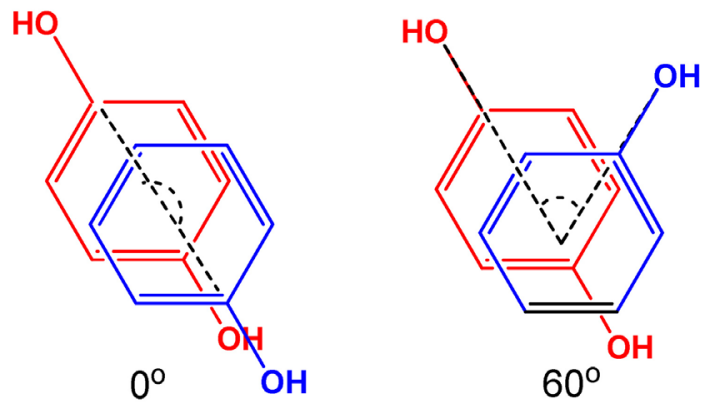

(c)

Figure 2. (a) Pictorial diagrams showing different dihedral angles of phenol-phenol stacking. (b) Pictorial diagrams showing different dihedral angles of diphenol-diphenol stacking. (c) Pictorial diagrams showing different dihedral angles of phenol-diphenol stacking.

Same procedure was carried out to construct the stacked models for Diphenol-Diphenol and Phenol-Diphenol stacking. We have carried out all computation calculations by DFT method using B3LPY/6-311++G(d,p) and 


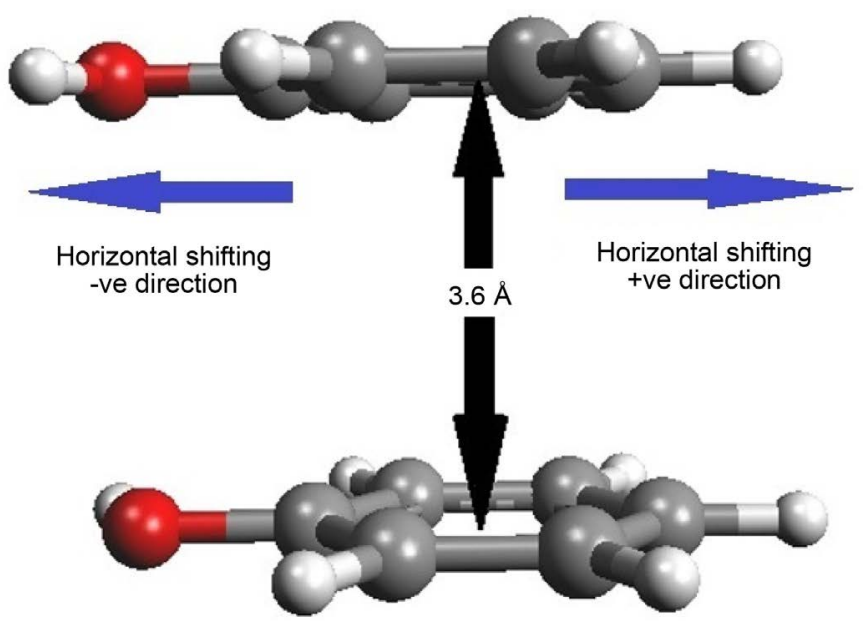

Figure 3. Figure depicting the intermolecular separation and horizontal shifting between two stacked monomers.

M062X/6-311++G(d,p) basis set, but M062X is more reliable to calculate stacking interaction energy values as it gives more negative values. During the construction of stacked phenol-phenol model, one phenol ring has been horizontally shifting along $\mathrm{X}, \mathrm{Y}$ or $\mathrm{Z}$-axis (from positive to negative end), keeping the other ring at constant position. This process was carried out to get the most favored minimized stacked model with minimum repulsion as well as the highly repulsive stacked model. In this case, the horizontal shifting for the stacked model was investigated along $\mathrm{X}$-axis from -3 to $+3 \AA$. Similarly, all the staggered conformations for the stacked models of phenol-phenol stacking systems were also prepared with dihedral angles $60^{\circ}, 120^{\circ}$ and $180^{\circ}$ respectively. Same procedure was carried out to construct the stacked models for Diphenol-Diphenol and Phenol-Diphenol stacking. We have carried out all computation calculations by DFT method using B3LPY/6-311++G(d,p) and M062X/6-311++G(d,p) basis set, but M062X is more reliable to calculate stacking interaction energy values as it gives more negative values.

The direction of $-\mathrm{OH}$ group of the phenolic ring in a stacked model always makes a significant impact on the overall stability of any stacked phenolic system. The $-\mathrm{OH}$ groups of a phenolic stacked model, with two phenolic rings may be either facing in same direction or opposite direction as shown in Figure 4. To investigate the accurate and effective stacking interaction in phenolic dimers, we have also carefully investigated the direction of $-\mathrm{OH}$ group of the two phenolic ring within a staked model. The change in direction of $-\mathrm{OH}$ group of a phenol ring in a stacked phenolic dimer also observes in stacking interaction energies, phenol-phenol stacking interaction becomes more favoured when -OH group of two phenol rings are facing opposite to each other.

The relative changes for the $\pi-\pi$ stacking interaction energies in gas phase, with M062X methods for different stacked phenolic systems are shown in Table 1 and certain stable structures are located from the local minima in the potential energy curves shown in Figures 5-7. The more negative interaction energy value 


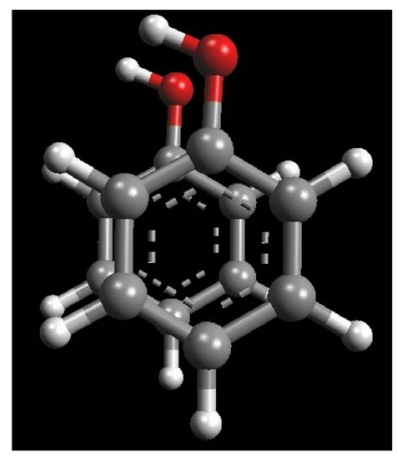

(a)

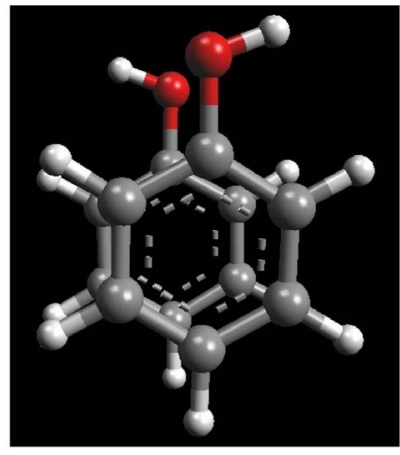

(b)

Figure 4. Stacked models for (a) Phenol-Phenol stacking with -OH group facing the same side and (b) Phenol-Phenol stacking with $-\mathrm{OH}$ group facing away from each other.

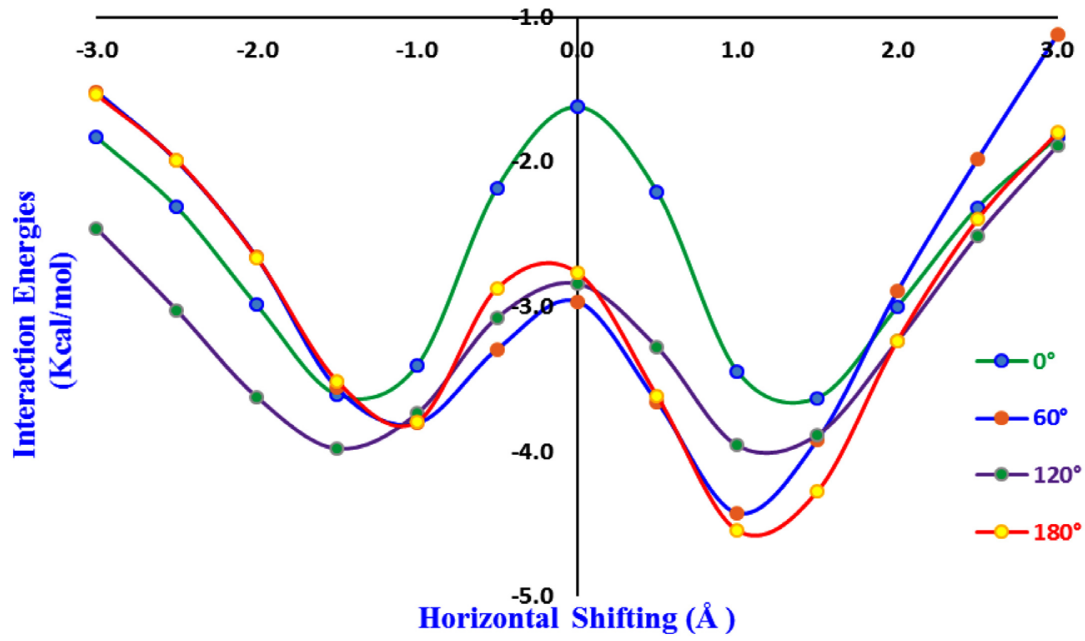

(a)

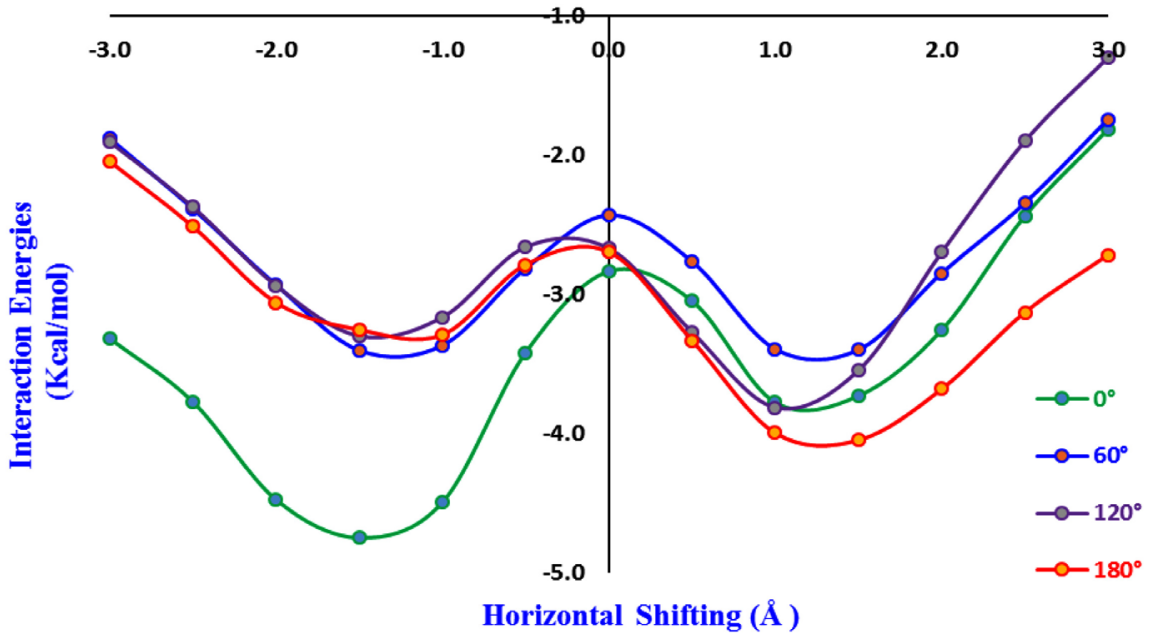

(b)

Figure 5. (a) Plot of interaction energies (MP2 single point) versus stacking positions ( $)$ for different conformations of phenol where the $-\mathrm{OH}$ groups are facing the same side. $(b)$ Plot of interaction energies (MP2 single point) versus stacking positions ( $\mathrm{A}$ ) for different conformations of phenol where the $-\mathrm{OH}$ groups are facing the opposite sides. 


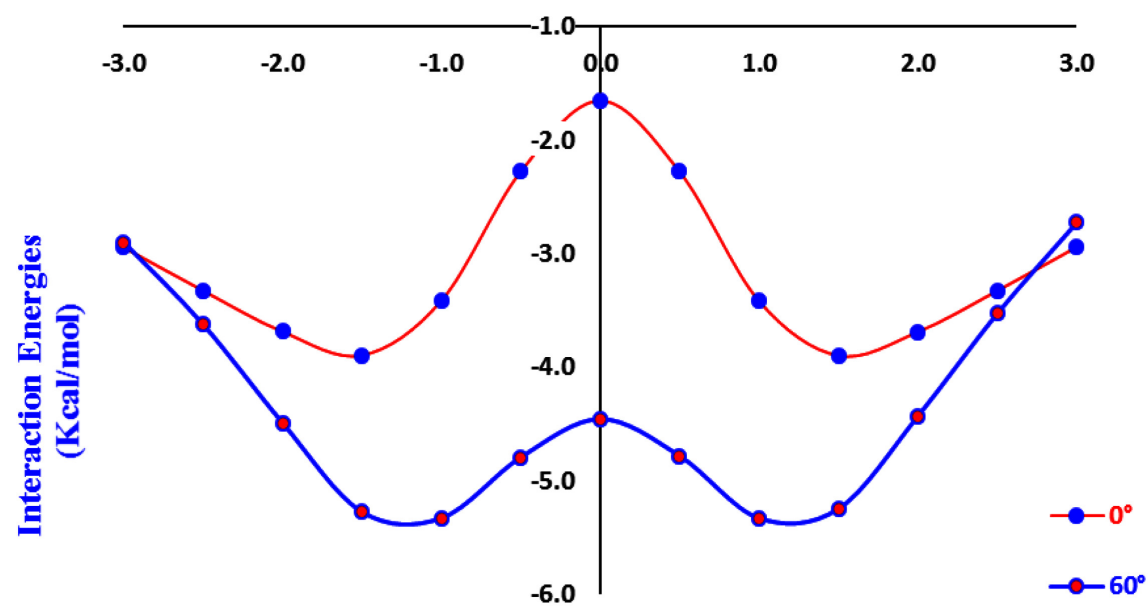

Horizontal Shifting $(\AA)$

(a)

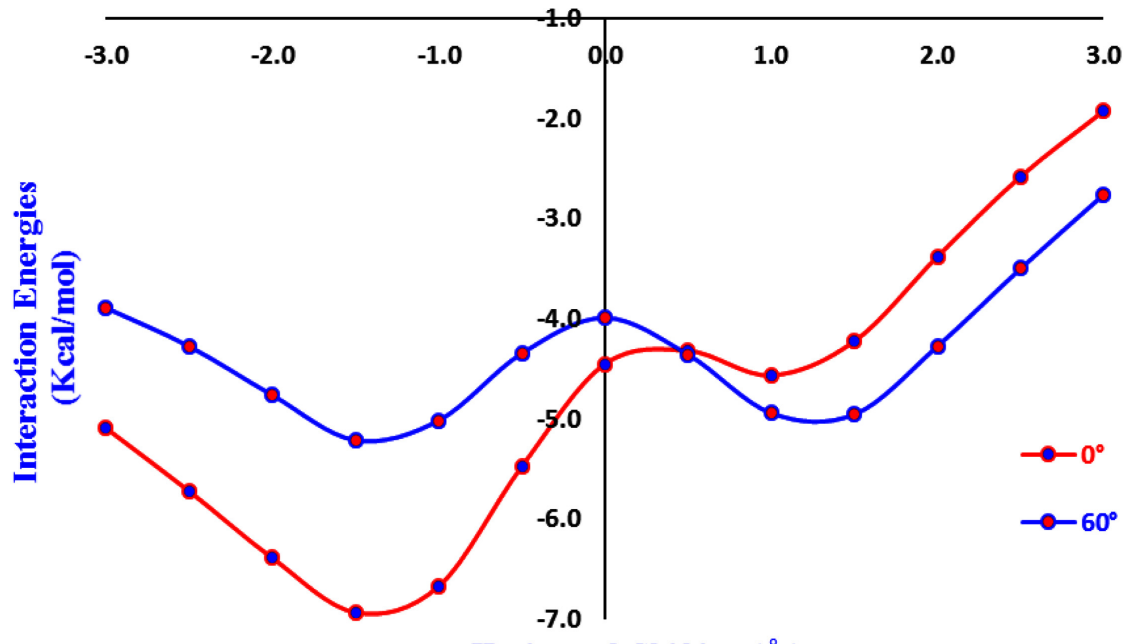

Horizontal Shifting $(\AA)$

(b)

Figure 6. (a) Plot of interaction energies (MP2 single point) versus stacking positions ( $)$ for different conformations of diphenol where the $-\mathrm{OH}$ groups are facing the same side. (b) Plot of interaction energies (MP2 single point) versus stacking positions ( $\AA$ ) for different conformations of diphenol where the $-\mathrm{OH}$ groups are facing the opposite sides.

or minima of the interaction energy plots results the more stable stacked model. Here, we can clearly see the differences in stacking interaction energies of phenolic systems with different dihedral angle as well as change in the direction of -OH groups of the stacked models (Figure 2 and Figure 4). In phenol-phenol $\pi-\pi$ stacking interaction, when the $-\mathrm{OH}$ groups of two phenol rings are facing each other in the same direction, then the sequence of stability of staggered stacked conformations are found as $0^{\circ}<120^{\circ}<60^{\circ}<180^{\circ}$. The most stable conformation among the different stacked conformations are found at a dihedral angle of $180^{\circ}$, where one phenol ring is rotating up to 180 intermolecular rotation to place itself completely opposite to the other phenol ring resulting in 


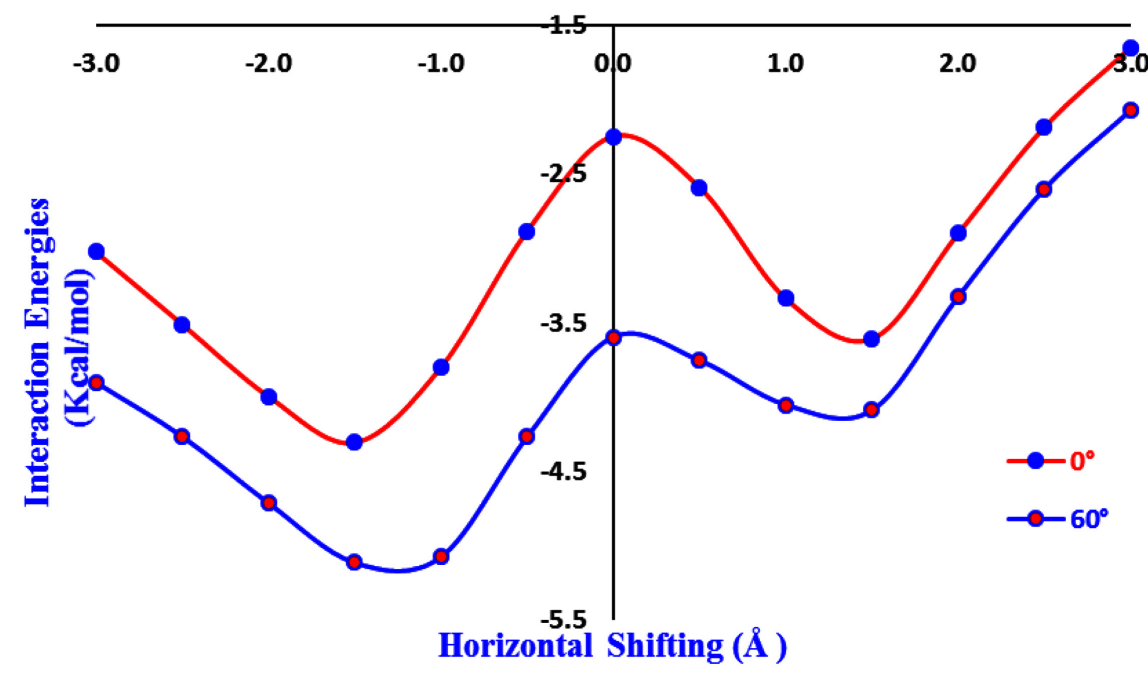

(a)

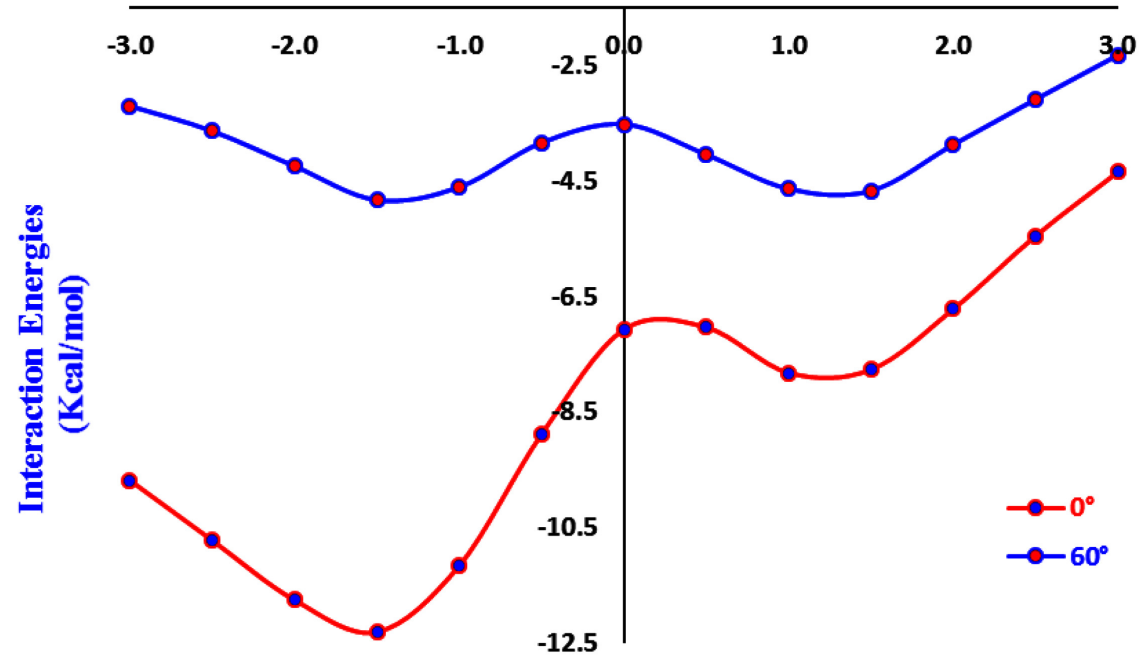

Horizontal Shifting ( $(\AA)$

(b)

Figure 7. (a) Plot of interaction energies (MP2 single point) versus stacking positions ( $)$ for different conformations of phenol-diphenol where the $-\mathrm{OH}$ groups are facing the same side. (b) Plot of interaction energies (MP2 single point) versus stacking positions ( $\mathrm{A})$ for different conformations of phenol-diphenol where the $-\mathrm{OH}$ groups are facing the opposite sides.

minimum repulsion (Figure 8). The interaction energy of this minimized stacked model was computed as $-4.5439 \mathrm{kcal} / \mathrm{mol}$ at a horizontal shifting of +1.0 $\AA$ (Table 1). Moreover, we also carried out the same procedure, where the $-\mathrm{OH}$ groups of the stacked phenol rings facing opposite to one another for all the stacked models, then the eclipsed stacked conformers with dihedral angle $0^{\circ}$ gets effectively stabilized than other conformations (Figure 9). Here, the sequence of stability of stacked models are found as $60^{\circ}<120^{\circ}<180^{\circ}<0^{\circ}$. The interaction energy of minimized stacked model was computed as $-4.7468 \mathrm{kcal} / \mathrm{mol}$ at a horizontal shifting of $-1.5 \AA$ (Table 1 ). 
Table 1. Stacking interaction energies $(\mathrm{kcal} / \mathrm{mol})$ for minimized phenolic stacked models. (M062X/6-311++G(d,p)).

\begin{tabular}{cccc}
\hline \multirow{2}{*}{ Stacked Models } & Dihedral Angles $(\varphi)$ & \multicolumn{2}{c}{ Interaction Energies (kcal/mol) } \\
\cline { 3 - 4 } & $0^{\circ}$ & - OH same side & - OH opposite side \\
\hline Phenol-Phenol Stacking & $60^{\circ}$ & -3.6292 & -4.7468 \\
& $120^{\circ}$ & -4.4238 & -3.3999 \\
Diphenol-Diphenol & $180^{\circ}$ & -3.9786 & -3.8103 \\
Stacking & $0^{\circ}$ & -4.5439 & -4.0456 \\
Phenol-Diphenol & $60^{\circ}$ & -3.8986 & -6.9298 \\
Stacking & $0^{\circ}$ & -5.3295 & -5.2118 \\
& $60^{\circ}$ & -4.3055 & -12.3070 \\
& & -5.1123 & -4.8308 \\
\hline
\end{tabular}

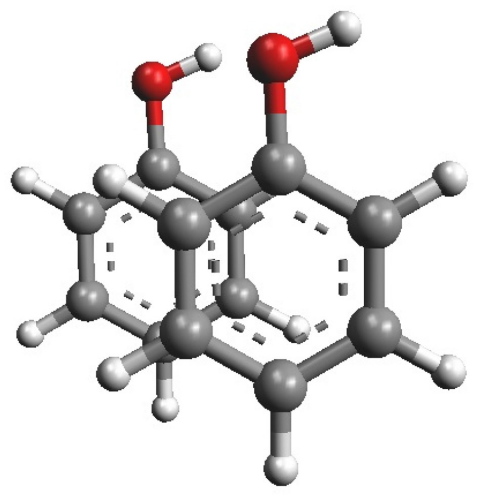

(a)

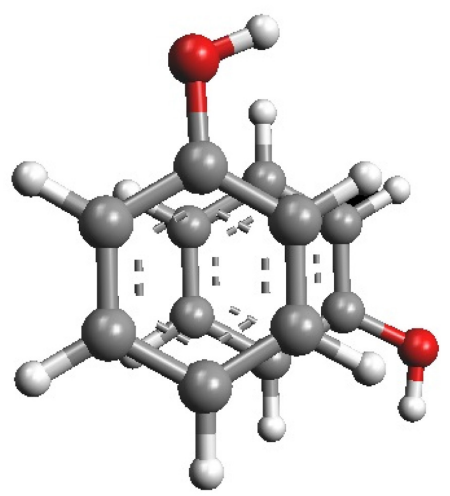

(c)

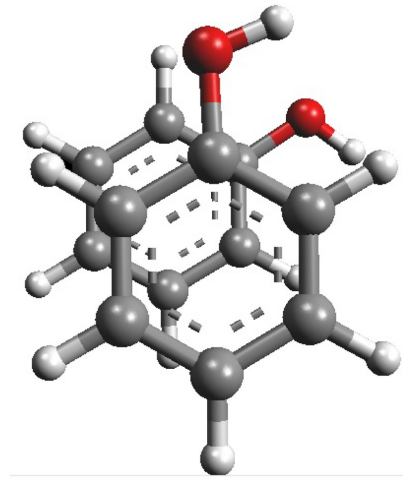

(b)

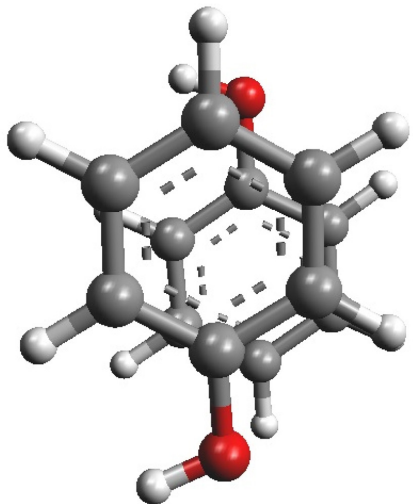

(d)

Figure 8. Minimized stable stacked models of Phenol-Phenol dimer with $-\mathrm{OH}$ groups of the phenol ring facing the same side in gas phase; (a) $0^{\circ}$ (b) $60^{\circ}$ (c) $120^{\circ}$ (d) $180^{\circ}$.

Similar procedure was followed to calculate the stacking interaction energies for diphenol-diphenol and phenol-diphenol stacked system. In these systems, interaction energies of the stacked models with dihedral angles $0^{\circ}$ and $60^{\circ}$ have only been computed as $0^{\circ}-180^{\circ}$ and $60^{\circ}-120^{\circ}$. For diphenol-diphenol and phenol-diphenol systems, where the $-\mathrm{OH}$ group of the two phenol rings facing 


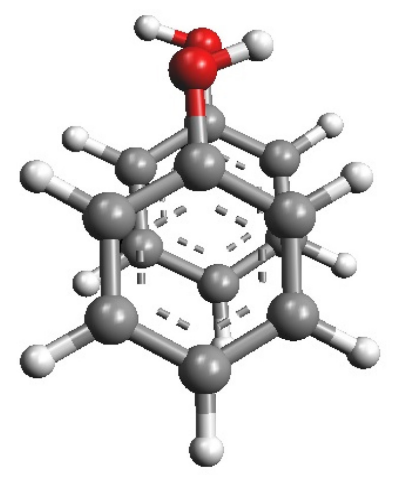

(a)

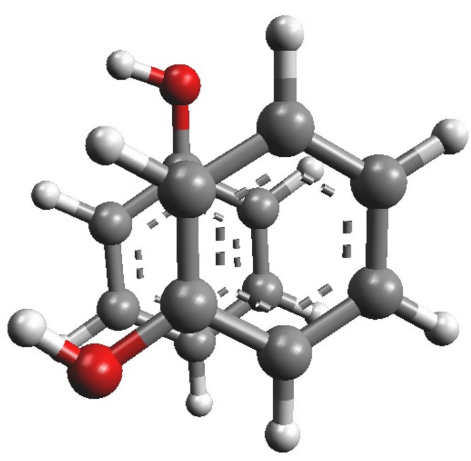

(c)

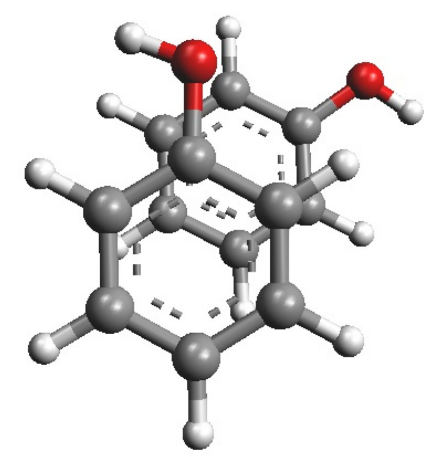

(b)

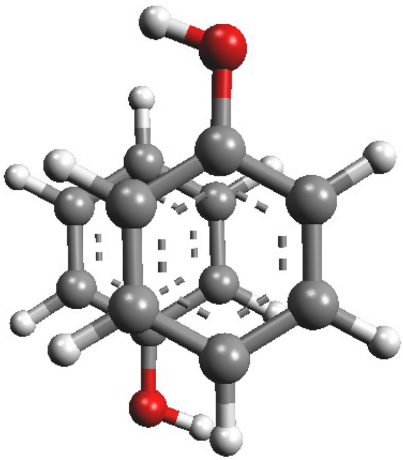

(d)

Figure 9. Minimized stable stacked models of Phenol-Phenol dimer with $-\mathrm{OH}$ groups of the phenol ring facing away from each other in gas phase; (a) $0^{\circ}$ (b) $60^{\circ}$ (c) $120^{\circ}$ (d) $180^{\circ}$.

each other, stacking interactions for $0^{\circ}$ conformation is found to be more stable than that of $60^{\circ}$ conformations. The interaction energies of minimized stacked model for diphenol diphenol and phenol-diphenol systems were computed as -5.3295 and $-5.1123 \mathrm{kcal} / \mathrm{mol}$ respectively. On the other hand, when the $-\mathrm{OH}$ groups of the two phenol rings face opposite to one another, the stacked systems with dihedral angle $0^{\circ}$ is much more stable than $60^{\circ}$ conformations. The interaction energies are also found to be more negative than that of other conformers, the minimized stacked interaction energy values for diphenol-diphenol and phenol diphenol stacked models were computed as -6.9298 and -12.3070 $\mathrm{kcal} / \mathrm{mol}$ respectively. Generally, in any stacked model of phenolic system at $0^{\circ}$ intermolecular rotation (i.e. $0^{\circ}$ dihedral angle) the stacked models are found to be more repulsive due to the strong repulsion between the $-\mathrm{OH}$ groups of the phenol rings. But, in this investigation it has been observed that when the $-\mathrm{OH}$ of the two phenol rings of the stacked models are facing opposite to each other then $0^{\circ}$ conformation can also give the much more stable stacked models (Figures 10-13). To observe the effective change in stacking interaction energies within the phenolic dimer, we can also compare the Mullikan charges density of -OH groups for unstacked and stacked phenolic systems, Mullikan charge density shows a significant change for minimized stacked models as compared to the unstacked model (Tables 2-4). 


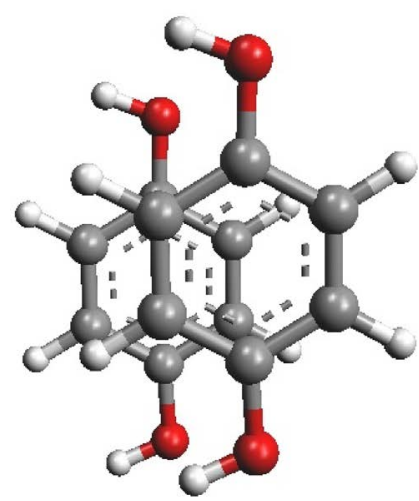

(a)

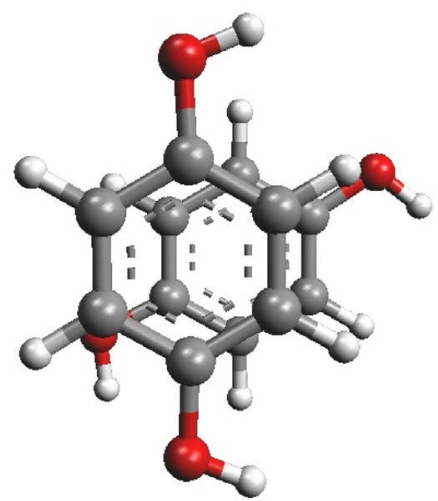

(b)

Figure 10. Minimized stable stacked models of Diphenol-Diphenol dimer with $-\mathrm{OH}$ groups of the diphenol ring facing the same side in gas phase; (a) $0^{\circ}$, (b) $60^{\circ}$.

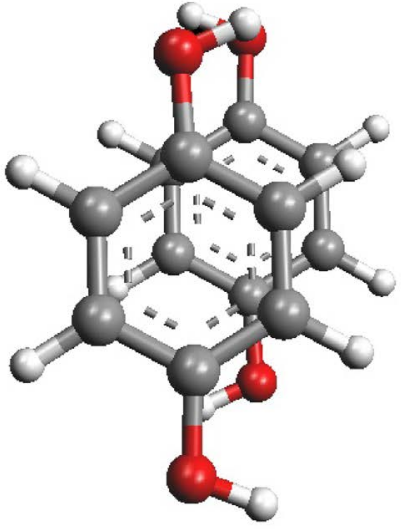

(a)

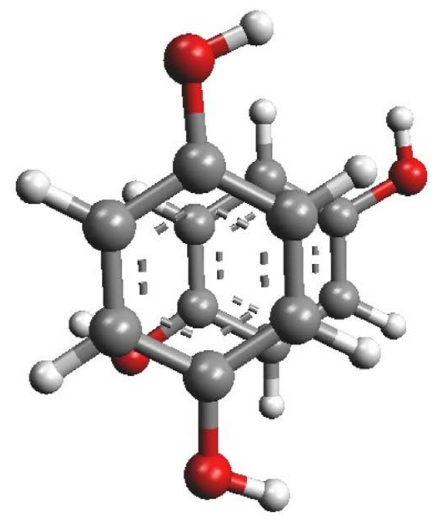

(b)

Figure 11. Minimized stable stacked models of Diphenol-Diphenol dimer with $-\mathrm{OH}$ groups of the diphenol ring facing away from each other in gas phase; (a) $0^{\circ}$, (b) $60^{\circ}$.

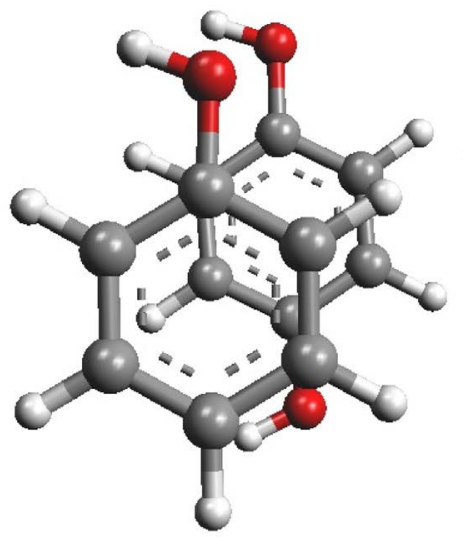

(a)

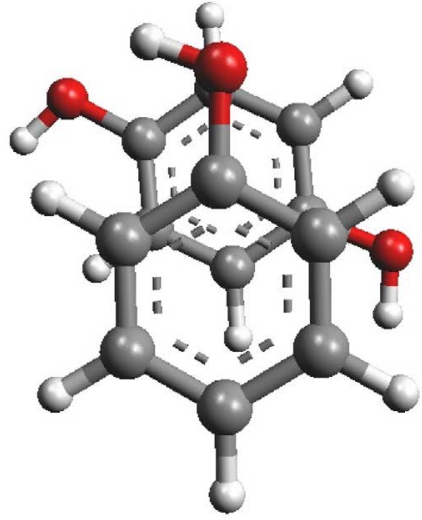

(b)

Figure 12. Minimized stable stacked models of Phenol-Diphenol dimer with $-\mathrm{OH}$ groups of the Phenol-Diphenol ring facing the same side in gas phase; (a) $0^{\circ}$, (b) $60^{\circ}$. 


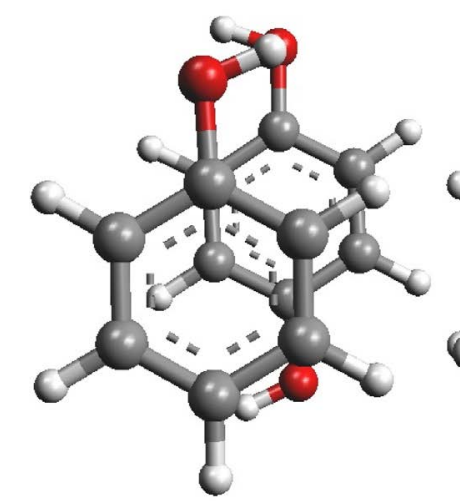

(a)

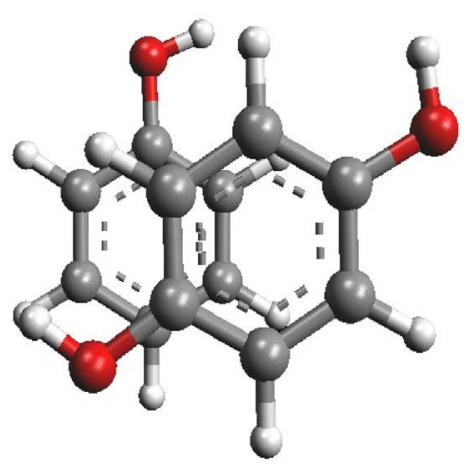

(b)

Figure 13. Minimized stable stacked models of Phenol-Diphenol dimer with $-\mathrm{OH}$ groups of the Phenol-diphenol ring facing away from each other in gas phase; (a) $0^{\circ}$, (b) $60^{\circ}$.

Table 2. Computed mulliken charges forminimized phenol-phenol stacked models for different dihedral angles with $-\mathrm{OH}$ groups are same and opposite direction $(\mathrm{M} 062 \mathrm{X} / 6-311++\mathrm{G}(\mathrm{d}, \mathrm{p}) \mathrm{POP}=\mathrm{NPA})$.

\begin{tabular}{|c|c|c|c|c|c|c|c|}
\hline \multirow{4}{*}{$\begin{array}{l}\text { Stacked } \\
\text { Models }\end{array}$} & \multirow{4}{*}{$\begin{array}{c}\text { Dihedral } \\
\text { Angles }(\varphi)\end{array}$} & \multicolumn{6}{|c|}{ Mulliken Charges (kcal/mol) } \\
\hline & & \multirow{2}{*}{\multicolumn{2}{|c|}{ Unstacked Phenol }} & \multicolumn{4}{|c|}{ Stacked Phenol } \\
\hline & & & & \multicolumn{2}{|c|}{-OH of Ring1 } & \multicolumn{2}{|c|}{$-\mathrm{OH}$ of Ring2 } \\
\hline & & $\mathrm{O}$ & $\mathrm{H}$ & $\mathrm{O}$ & $\mathrm{H}$ & $\mathrm{O}$ & $\mathrm{H}$ \\
\hline \multirow{4}{*}{$\begin{array}{c}\text {-OH Same } \\
\text { direction }\end{array}$} & $0^{\circ}$ & -0.2370 & 0.2650 & -0.2094 & 0.2726 & -0.2105 & 0.2669 \\
\hline & $60^{\circ}$ & -0.2370 & 0.2650 & -0.2240 & 0.2698 & -0.2095 & 0.2681 \\
\hline & $120^{\circ}$ & -0.2370 & 0.2650 & -0.2190 & 0.2683 & -0.2168 & 0.2720 \\
\hline & $180^{\circ}$ & -0.2370 & 0.2650 & -0.2030 & 0.2598 & -0.2022 & 0.2596 \\
\hline \multirow{4}{*}{$\begin{array}{c}-\mathrm{OH} \\
\text { opposite } \\
\text { direction }\end{array}$} & $0^{\circ}$ & -0.2370 & 0.2650 & -0.2135 & 0.2711 & -0.1950 & 0.2659 \\
\hline & $60^{\circ}$ & -0.2370 & 0.2650 & -0.2198 & 0.2678 & -0.1896 & 0.2577 \\
\hline & $120^{\circ}$ & -0.2370 & 0.2650 & -0.2060 & 0.2616 & -0.2049 & 0.2608 \\
\hline & $180^{\circ}$ & -0.2370 & 0.2650 & -0.1957 & 0.2608 & -0.1942 & 0.2591 \\
\hline
\end{tabular}

Table 3. Computed mulliken charges for minimized diphenol-diphenol stacked models for different dihedral angles with $-\mathrm{OH}$ groups are same and opposite direction $(\mathrm{M} 062 \mathrm{X} / 6-311++\mathrm{G}(\mathrm{d}, \mathrm{p}) \mathrm{POP}=\mathrm{NPA})$.

\begin{tabular}{|c|c|c|c|c|c|c|c|c|c|}
\hline \multirow{3}{*}{$\begin{array}{l}\text { Stacked } \\
\text { Models }\end{array}$} & \multirow{3}{*}{$\begin{array}{c}\text { Dihedral } \\
\text { Angles }(\varphi)\end{array}$} & \multicolumn{8}{|c|}{ Mulliken Charges of Stacked Diphenol } \\
\hline & & \multicolumn{4}{|c|}{-OH of Ring1 } & \multicolumn{4}{|c|}{$-\mathrm{OH}$ of Ring2 } \\
\hline & & $\mathrm{O}$ & $\mathrm{H}$ & $\mathrm{O}$ & $\mathrm{H}$ & $\mathrm{O}$ & $\mathrm{H}$ & $\mathrm{O}$ & $\mathrm{H}$ \\
\hline \multirow{2}{*}{$\begin{array}{c}\text {-OH same } \\
\text { direction }\end{array}$} & $0^{\circ}$ & -0.2113 & 0.2632 & -0.2094 & 0.2661 & -0.2010 & 0.2635 & -0.2302 & 0.2665 \\
\hline & $60^{\circ}$ & -0.2236 & 0.2686 & -0.2194 & 0.2690 & -0.2281 & 0.2722 & -0.2307 & 0.2671 \\
\hline $\begin{array}{c}-\mathrm{OH} \\
\text { opposite }\end{array}$ & $0^{\circ}$ & -0.2269 & 0.2749 & -0.2175 & 0.2720 & -0.2178 & 0.2722 & -0.2266 & 0.2748 \\
\hline direction & $60^{\circ}$ & -0.2289 & 0.2674 & -0.2035 & 0.2611 & -0.2272 & 0.2701 & -0.2168 & 0.2690 \\
\hline
\end{tabular}

${ }^{*} \mathrm{MC}$ for unstacked diphenol: $\mathrm{O}=-0.2494$ and $\mathrm{H}=0.2640$. 
Table 4. Computed mulliken charges for minimized phenol-diphenol stacked models for different dihedral angles with $-\mathrm{OH}$ groups are same and opposite direction $(\mathrm{M} 062 \mathrm{X} / 6-311++\mathrm{G}(\mathrm{d}, \mathrm{p}) \mathrm{POP}=\mathrm{NPA})$.

\begin{tabular}{|c|c|c|c|c|c|c|c|}
\hline \multirow{3}{*}{$\begin{array}{l}\text { Stacked } \\
\text { Models }\end{array}$} & \multirow{3}{*}{$\begin{array}{c}\text { Dihedral } \\
\text { Angles } \\
(\varphi)\end{array}$} & \multicolumn{6}{|c|}{ Mulliken Charges of Stacked Phenol-Diphenol system } \\
\hline & & \multicolumn{2}{|c|}{$\begin{array}{c}-\mathrm{OH} \text { of Ring of } \\
\text { phenol }\end{array}$} & \multicolumn{4}{|c|}{$-\mathrm{OH}$ of Ring of diphenol } \\
\hline & & $\mathrm{O}$ & $\mathrm{H}$ & $\mathrm{O}$ & $\mathrm{H}$ & $\mathrm{O}$ & $\mathrm{H}$ \\
\hline $\begin{array}{l}-\mathrm{OH} \\
\text { same }\end{array}$ & $0^{\circ}$ & -0.1971 & 0.2646 & -0.2310 & 0.2668 & -0.2173 & 0.2635 \\
\hline direction & $60^{\circ}$ & -0.1850 & 0.2603 & -0.2305 & 0.2689 & -0.2125 & 0.2629 \\
\hline $\begin{array}{c}-\mathrm{OH} \\
\text { opposite }\end{array}$ & $0^{\circ}$ & -0.2049 & 0.2724 & -0.2267 & 0.2736 & -0.2178 & 0.2638 \\
\hline direction & $60^{\circ}$ & -0.2187 & 0.2719 & -0.2273 & 0.2655 & -0.2061 & 0.2584 \\
\hline
\end{tabular}

${ }^{*} \mathrm{MC}$ for unstacked diphenol: $\mathrm{O}=-0.2494, \mathrm{H}=0.2640$ and Phenol $\mathrm{O}=-0.2370, \mathrm{H}=0.2650$.

\section{Conclusion}

From the above investigations, it has been observed that the phenolic systems are well stacked within themselves. But, the intermolecular rotation and direction of -OH groups of the phenolic systems play an important role in the stability of the stacked models. We can conclude that when the $-\mathrm{OH}$ groups of the stacked models are facing same direction then $180^{\circ}$ or $60^{\circ}$ conformations gets more stabilized. Whereas, when the $-\mathrm{OH}$ groups are facing opposite to one another then the eclipsed conformation with $0^{\circ}$ dihedral angle gives more stable stacked model.

\section{Acknowledgements}

Authors are highly grateful to the AICTE-TEQIP-3 fund and Ministry of Human Resource Development (MHRD), New Delhi, for providing research assistance.

\section{Conflicts of Interest}

The authors declare no conflicts of interest regarding the publication of this paper.

\section{References}

[1] Kool, E.T., Morales, J.C. and Guckian, K.M. (2000) Mimicking the Structure and Function of DNA: Insights into DNA Stability and Replication. Angewandte Chemie, 39, 900-1009. https://doi.org/10.1002/(SICI)1521-3773(20000317)39:6<990::AID-ANIE990>3.0.C $\mathrm{O} ; 2-0$

[2] Hunter, C.A., Lawson, K.R., Perkins, J. and Urch, C.J. (2001) Aromatic Interactions. Journal of the Chemical Society, Perkin Transactions 2, 5, 651-669. https://doi.org/10.1039/b008495f

[3] Waters, M.L. (2002) Aromatic Interactions in Model Systems. Current Opinion in Chemical Biology, 6, 736-741. https://doi.org/10.1016/S1367-5931(02)00359-9

[4] Meyer, E.A., Castellano, R.K. and Diederich, F. (2003) Interactions with Aromatic 
Rings in Chemical and Biological Recognition. Angewandte Chemie, 42, 1210-1250. https://onlinelibrary.wiley.com/doi/pdf/10.1002/anie.200390319 https://doi.org/10.1002/anie.200390319

[5] Sarkhel, S., Rich, A. and Egli, M. (2003) Water-Nucleobase "Stacking": H- $\pi$ and Lone Pair- $\pi$ Interactions in the Atomic Resolution Crystal Structure of an RNA Pseudoknot. Journal of American Chemical. Society, 125, 8998-8999. https://doi.org/10.1021/ja0357801

[6] Coates, G.W., Dunn, A.R., Henling, L.M., Dougherty, D.A. and Grubbs, R.H. (1997) Phenyl-Perfluorophenyl Stacking Interactions: A New Strategy for Supermolecule Construction. Angewandte Chemie, 36, 248-251.

https://doi.org/10.1002/anie.199702481

[7] Suzuki, M., Amano, N., kakinuma, J. and Tateno, M. (1997) Use of a 3D Structure Data Base for Understanding Sequence-Dependent Conformational Aspects of DNA. Journal of Molecular Biology, 274, 421-435.

https://doi.org/10.1006/jmbi.1997.1406

[8] Mathews, D.H, Sabina, J., Zuker, M. and Turner, D.H. (1999) Expanded Sequence Dependence of Thermodynamic Parameters Improves Prediction of RNA Secondary Structure. Journal of Molecular Biology, 288, 911-970.

https://doi.org/10.1006/jmbi.1999.2700

[9] Bommarito, S., Peyret, N. and Santa Lucia Jr., J. (2000) Thermodynamic Parameters for DNA Sequences with Dangling Ends. Nucleic Acids Research, 28, 1929-1934. https://doi.org/10.1093/nar/28.9.1929

[10] Di Fenza, A., Heine, A., Koert, U. and Klebe, G. (2007) Understanding Binding Selectivity toward Trypsin and Factor Xa: The Role of Aromatic Interactions. ChemMedChem, 2, 297-308. https://doi.org/10.1002/cmdc.200600185

[11] Georgia, B.M., Marc, G. and Anthony, K.R. (1998) $\pi$-Stacking Interactions: ALIVE AND WELL IN PROTEINS. Journal of Biological Chemistry, 273, 15458-15463. https://doi.org/10.1074/jbc.273.25.15458

[12] Sal-Man, N., Gerber, D., Bloch, I. and Slai, Y. (2007) Specificity in Transmembrane Helix-Helix Interactions Mediated by Aromatic Residues. Journal of Biological Chemistry, 282, 19753-19761. https://doi.org/10.1074/jbc.M610368200

[13] Cozzi, F., Ponzini, F., Annunziata, R., Cinquini, M. and Siegel, J.S. (1995) Polar Interaction between Stacked $\pi$ Systems in Flourinated 1,8-Diarylnapthalenes: Imortance of Quardruple Moments in Molecular Recognition. Angewandte Chemie, 34, 1019-1020. https://doi.org/10.1002/anie.199510191

[14] Mark, J.R. and Marcey, L.W. (2002) Unexpected Substituent Effects in Offset $\pi-\pi$ Stacked Interactions in Water. Journal of the American Chemical Society, 124, 1860-1862. https://doi.org/10.1021/ja016508z

[15] Zhao, Y. and Truhlar, D. G. (2005) Benchmark Databases for Nonbonded Interactions and Their Use to Test Density Functional Theory. Journal of Chemical Theory and Computation, 1, 415-432. https://doi.org/10.1021/ct049851d

[16] Yi, M. and Scheiner, S. (1996) Proton Transfer in the $\left[\right.$ Phenol- $\left.\mathrm{NH}_{3}\right]+$ System: An Experimental and $A b$ initio Study. Chemical Physics Letters, 262, 567. https://doi.org/10.1016/S0009-2614(96)01135-9

[17] Brutschy, B. (1992) Ion-Molecule Reactions within Molecular Clusters. Chemical Reviews, 92, 1567-1587. https://doi.org/10.1021/cr00015a005

[18] Dopfer, O., Reiser, G., Muller-Dethlafs, K., Schlag, E.W. and Colson, S.D. (1994) Watching Proton Transfer in Real Time: Ultrafast Photoionization-Induced Proton 
Transfer in Phenol Ammonia Complex Cation. The Journal of Chemical Physics, 101, 974. https://doi.org/10.1063/1.467752

[19] Lipert, R.J. and Colson, S.D. (1988) Study of Phenol-Water Complexes Using Frequency- and Time-Resolved Pump-Probe Photoionization. The Journal of Chemical Physics, 89, 4579-4585. https://doi.org/10.1063/1.454798

[20] Lipert, R.J. and Colson, S.D. (1989) Deuterium Isotope Effects on S1 Radiationless Decay in Phenol and on Intermolecular Vibrations in the Phenol-Water Complex. Journal of Physical Chemistry, 93, 135-139. https://doi.org/10.1021/j100338a030

[21] Korenaga, T., Shoji, T., Onoue, K. and Sakai, T. (2009) Demonstration of the Existence of Intermolecular Lone Pair $\cdots \pi$ Interaction between Alcoholic Oxygen and the $\mathrm{C}_{6} \mathrm{~F}_{5}$ Group in Organic Solvent. Chemical Communications, 4678-4680. https://doi.org/10.1039/b908752d

[22] Alkorta, I., Rozas, I. and Elguero, J. (1997) An Attractive Interaction between the p-Cloud of $\mathrm{C}_{6} \mathrm{~F}_{6}$ and Electron-Donor Atoms. Journal of Organic Chemistry, 62, 4687-4691. https://doi.org/10.1021/jo970125v

[23] Sinnokrot, M.O., Valeev, E.F. and Sherrill, C.D. (2002) Estimates of the Ab Initio Limit for $\pi-\pi$ Interactions: The Benzene Dimer. Journal of American Chemical Society, 124, 10887-10893. https://doi.org/10.1021/ja025896h

[24] Sinnokrot, M.O. and Sherill, C.D. (2004) Highly Accurate Coupled Cluster Potential Energy Curves for the Benzene Dimer: Sandwich, T-Shaped, and Parallel-Displaced Configurations. Journal of Physical Chemistry A, 108, 10200-10207. https://doi.org/10.1021/jp0469517

[25] Parthasarathi, R., Subramanian, V. and Sathyamurthy, N. (2005) Hydrogen Bonding in Phenol, Water, and Phenol-Water Clusters. Journal of Physical Chemistry A, 109, 843-850. https://doi.org/10.1021/jp046499r

[26] Monteiro, C.J.P., Pereira, M.M., Azenha, M.E. Burrows, H.D., Serpa, C., Arnaut, L.G., Tapia, M.J., Sarakha, M.P., Chung, W.W. and Navaratnam, S. (2005) A Comparative Study of Water Soluble 5,10,15,20-tetrakis(2,6-dichloro-3-sulfophenyl) Porphyrin and Its Metal Complexes as Efficient Sensitizers for Photodegradation of Phenols. Photochemical \& Photobiological Sciences, 4, 617-624. https://doi.org/10.1039/b507597a

[27] Muller-Dethlefs, K. and Hobza, P. (2000) Noncovalent Interactions: A Challenge for Experiment and Theory. Chemical Reviews, 100, 143-168. https://doi.org/10.1021/cr9900331

[28] Skotheim, T.A., Reynolds, J.R. and Elsenbaumer, R.L. (1997) Hand Book of Conducting Polymer. 2nd Edition, Marcel Dekker, New York.

[29] Nalwa, H.S. (1997) Hand Book of Organic Conductive Molecules and Polymer Vol. 1-4. Wiley, New York.

[30] Frisch, M.J., Trucks, G.W., Schlegel, H.B., Gill, P.M.W., Johnson, B.G., Robb, M.A., Cheeseman, J.R., Keith, T., Petersson, G.A., Montgomery, J.A., Raghavachari, K., Al-Laham, M.A., Zakrzewaki, V.G., Ortiz, J.V., Foresmann, J.B., Ciolowski, J., Stefanov, B.B., Namayakkara, A., Challacombe, M., Peng, C.Y., Ayala, P.Y., Chen, W., Wong, M.W., Andres, J.L., Replogle, E.S., Gomperts, R., Martin, R.L., Fox, D.J., Binkley, J.S., Defrees, D.J., Baker, J., Stewart, J.P., Head-Gordon, M., Gonzalez, C. and Pople, J.A. (2009) Gaussian 09. Gaussian Inc., Pittsburgh. 